\title{
Enabling QKD under strong turbulence for wireless networks with tilt wavefront correction
}

\author{
Pablo Arteaga-Díaz, Alejandro Ocampos-Guillén, Veronica Fernandez* \\ Group of Cryptology and Information Security \\ Department of Information and Communication Technologies \\ Institute of Physical and Information Technologies (ITEFI), C/Serrano, 144, 28006, Madrid \\ Spanish National Research Council (CSIC) \\ Tel*: (0034) 915618806,e-mail*veronica.fernandez@iec.csic.es
}

\begin{abstract}
Wavefront tilt correction is necessary for reducing beam deviations caused by atmospheric turbulence in the receiver of an optical system. This reduction allows decreasing the receiver's field of view, which in the case of free-space quantum key distribution (QKD), reduces the solar background noise that reaches the detectors, and with this, the quantum bit error rate (QBER). A wavefront tilt correcting system that stabilizes the beam in two different points of the receiver's optical axis has been developed and characterised, and it is capable of operating under realistic conditions of strong turbulence with almost perfect ideal correction. The reduced area of beam deviations at the focal plane of the receiver after correction enables a reduction of the QBER of more than $80 \%$, which paves the way to free-space quantum key distribution in daylight under strong turbulence.
\end{abstract}

Keywords: free space quantum key distribution, beam tracking, wavefront tilt correction, quantum communications, beam steering.

\section{INTRODUCTION}

Future wireless networks will presumably be ubiquitous and will necessitate of two requirements. The first is high bandwidth which can be provided by optical signals, and the second is unconditional secure channels, which can be provided by Quantum Key Distribution (QKD). However, the atmosphere distorts the wavefront through several effects, which enlarge, deform and deviate the optical signals upon reception. Techniques for correcting these effects must therefore be applied, which can include solely wavefront tilt correction, the lowest order type of aberration, or also include correction of higher-order distortions, commonly referred to adaptive optics. However, in scenarios where many platforms will be mobile, lightweight and simple designs will be preferred whenever possible, and therefore it should be assessed whether correcting systems only including tilt compensation could be sufficient in certain scenarios, to enable a successful QKD link. We have worked on the implementation of a tilt correction system for a previously developed free-space polarization-encoding QKD system. We will present in this work recent results of the performance of said correcting system in mitigating atmospheric effects in a 300-meter free-space link between two buildings in Madrid, in moderate and strong turbulent regimes. We will determine the quality of the correction achieved compared to the theory and the effect on relevant parameters of a QKD system, such as the Quantum Bit Error Rate (QBER).

\section{WAVEFRONT DISTORTION THROUGH THE ATMOSPHERE}

Quantum communication is affected by the atmosphere, which produces local changes in temperature, causing refractive index fluctuations. These fluctuations affect a plane wavefront that crosses the atmosphere, introducing wavefront distortion. If the distortion implies a change in the angle of arrival of the beam at the receiver, represented by $\beta_{a}$, it is known as wavefront tilt, which in turn originates a phase difference $\Delta \phi$ such that:

$$
k \Delta l=\Delta \phi
$$

where $k$ is the wave number and $\Delta l$ the optical path difference. Assuming $\beta_{a}$ is sufficiently small so that $\beta_{a} \cong$ $\operatorname{sen}\left(\beta_{a}\right)$, we can use the following approximation of geometric optics:

$$
\beta_{a}=\frac{\Delta l}{D}=\frac{\Delta \phi}{k D}
$$

with $D$ as the aperture diameter of the optical system. This is the case when the long-term beam is equal or larger than the aperture. When the long-term beam is smaller, the maximum phase difference is limited by the maximum beam displacement, which is the long-term beam diameter. 


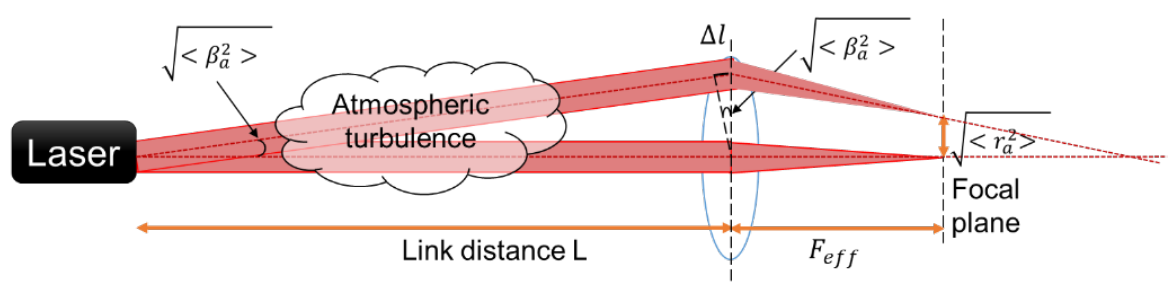

Figure 1. Angle of arrival caused by atmospheric turbulence and its effect on the focal plane of an optical receiver system.

Assuming $\left\langle\beta_{a}\right\rangle=0$, the variance of the angle of arrival is:

$$
\left\langle\beta_{a}{ }^{2}\right\rangle=\frac{\left\langle\Delta \phi^{2}\right\rangle}{(k D)^{2}}=\frac{D_{\phi}(D, L)}{(k D)^{2}}
$$

where $D_{\phi}(D, L)$ is the phase structure function [1]. It can be deduced then:

$$
D_{\phi}(D, L)=(k D)^{2}\left\langle\beta_{a}{ }^{2}\right\rangle
$$

Taking into account that under the Kolmogorov power spectral density of the refractive index and with an aperture $D$ in the range $\left(\sqrt{L / k} \ll D \ll L_{0}\right)$, being $L_{0}$ the outer scale, the phase structure function can be written as:

$$
D_{\phi}(D, L)=2.91 C_{n}^{2} k^{2} L D^{5 / 3}
$$

we arrive at:

$$
\left\langle\beta_{a}{ }^{2}\right\rangle=2.91 C_{n}^{2} L D^{-1 / 3}
$$

Using these equations and through the measurements of the angle of arrival we can infer the refractive structure parameter and characterized the turbulent regime.

\subsection{ANALYSIS OF THE WAVEFRONT DISTORTION THROUGH THE SERIES OF ZERNIKE MODIFIED POLYNOMIALS.}

The distortion of the phase wavefront, $\phi$, can be expressed as the sum of simple distortions, which can be modelled by the modified Zernike polynomials $Z_{i}[3]$ :

$$
\phi=\sum_{i=1}^{\infty} a_{i} Z_{i}
$$

where $a_{i}$ are the coefficients or 'weights' of each $Z_{i}$ that contribute to the total deformation of the beam. In practice, when some aberrations are corrected with the first $j$ Zernike functions, the quadratic residual error $\sigma_{J}^{2}$ is defined as the phase variance of the total phase $\left\langle\phi^{2}\right\rangle$ minus the phase variance of the total $j$ corrected components:

$$
\sigma_{J}^{2}=\left\langle\phi^{2}\right\rangle-\sum_{i=1}^{J}\left\langle\left|a_{i}\right|^{2}\right\rangle=\sum_{i=J+1}^{\infty}\left\langle\left|a_{i}\right|^{2}\right\rangle
$$

The solution is given in [2] by an expression that depends on the aperture of the optical system $D$ and the Fried correlation distance $r_{0}$, as:

$$
\sigma_{J}^{2}=\Delta_{J} \cdot\left(\frac{D}{r_{0}}\right)^{\frac{5}{3}} \quad\left[\mathrm{rad}^{2}\right]
$$

where $\Delta_{J}$ are constant values and $r_{0}$, for $z<<z_{R}$ has the following expression [1]:

$$
r_{0}=1.67\left(L k^{2} C_{n}^{2}\right)^{-3 / 5}
$$

$L$ is the distace the wave travels and $k$ the wavenumber. We have assumed $C_{n}^{2}$ stays constant through the distance, which can be assumed for horizontal paths. Substituting $r_{0}$ in equation (9) the following expression is obtained for the residual error:

$$
\sigma_{J}^{2}=(1.67)^{-5 / 3} \Delta_{J} C_{n}^{2} k^{2} L D^{5 / 3}
$$

\subsection{ESTIMATION OF THE QUALITY OF THE TILT CORRECTION}

We can evaluate the tilt correction achieved by comparing the theoretical and experimental values of the residual error. If the system is correcting tilt, then from equation (8), the residual error is of order three and has the form:

$$
\sigma_{3}^{2}=\left\langle\phi^{2}\right\rangle_{\text {total }}-\sum_{i=1}^{3}\left\langle\left|a_{i}\right|^{2}\right\rangle=\left\langle\phi^{2}\right\rangle_{\text {total }}-\left\langle\left|a_{1}\right|^{2}\right\rangle-\sum_{i=2}^{3}\left\langle\left|a_{i}\right|^{2}\right\rangle=\sigma_{1}^{2}-\sum_{i=2}^{3}\left\langle\left|a_{i}\right|^{2}\right\rangle=\sigma_{1}^{2}-\left\langle\phi^{2}(r)\right\rangle_{\text {tilt }} \text { (12) }
$$

where $\left\langle\phi^{2}(r)\right\rangle_{\text {tilt }}$ is the tilt phase variance, and therefore:

$$
\left\langle\phi^{2}(r)\right\rangle_{\text {tilt }}=\sigma_{1}^{2}-\sigma_{3}^{2}
$$

which using the formula (11) and knowing the values $\Delta_{1}=1.0266$ y $\Delta_{3}=0.134$ [2] we obtain:

$$
\left\langle\phi^{2}(r)\right\rangle_{\text {tilt }}=0.38 C_{n}^{2} k^{2} L D^{5 / 3}
$$

Equalling $C_{n}^{2} k^{2} L D^{5 / 3}$ from (5) and (14) we obtain the tilt phase variance: 


$$
\left\langle\phi^{2}(r)\right\rangle_{\text {tilt }}=\left(\frac{0.38}{2.91}\right) D_{\phi}(D, L)=0.13 D_{\phi}(D, L)=0.13(k D)^{2}\left\langle\beta_{a}{ }^{2}\right\rangle
$$

This last expression is total tilt variance. In practice, the correction is not perfect and the phase variance that we can correct is the difference between the total phase variance without correction and the phase variance after correcting:

$$
\left\langle\phi^{2}(r)\right\rangle_{t_{\text {till }} \text { corrected }}=\left\langle\phi^{2}(r)\right\rangle_{\text {tilt }}-\left\langle\phi^{2}(r)\right\rangle_{\text {till }_{\text {not_corrected }}}=0.13(k D)^{2}\left\langle\beta_{a_{-} \text {OFF }}{ }^{2}\right\rangle-0.13(k D)^{2}\left\langle\beta_{a_{-} \text {ON }}{ }^{2}\right\rangle
$$

where $\beta_{a_{-} \text {OFF }}$ and $\beta_{a_{O} \text { ON }}$ are the angle of arrival when the correcting system is OFF and ON, respectively. Therefore, it is possible to estimate the residual error of tilt correction from the expression:

$$
\sigma_{3}^{2}=\sigma_{1}^{2}-\left\langle\phi^{2}(r)\right\rangle_{\text {tilt_corrected }}=\sigma_{1}^{2}-0.13(k D)^{2}\left[\left\langle\beta_{a_{-} \text {OFF }}{ }^{2}\right\rangle-\left\langle\beta_{a_{-} \text {ON }}{ }^{2}\right\rangle\right]
$$

Since:

we obtain:

$$
\sigma_{1}^{2}=0.44 C_{n}^{2} k^{2} L D^{5 / 3}
$$

$$
\sigma_{3}^{2}=0.44 C_{n}^{2} k^{2} L D^{5 / 3}-0.13(k D)^{2}\left[\left\langle\beta_{a_{-} O F F}{ }^{2}\right\rangle-\left\langle\beta_{a_{-} O N}{ }^{2}\right\rangle\right]
$$

We also need to consider that we are correcting using a different wavelength from the quantum channel $(1550 \mathrm{~nm}$ instead of $850 \mathrm{~nm}$ ) and therefore we have an error associated to this difference. If in the expression (11) we set the order three for tilt correction we have:

$$
\sigma_{3}^{2}(\lambda)=0.057 C_{n}^{2} L D^{5 / 3}\left(\frac{2 \pi}{\lambda}\right)^{2}
$$

The ratio of this expression for both wavelengths is:

Adding this error to the residual error we have:

$$
\begin{gathered}
\frac{\sigma_{3}^{2}(850 \mathrm{~nm})}{\sigma_{3}^{2}(1550 \mathrm{~nm})}=\left(\frac{1550}{850}\right)^{2}=3.3253 \\
\sigma_{3}^{2}(850 \mathrm{~nm})=3.3253 \cdot \sigma_{3}^{2}(1550 \mathrm{~nm})
\end{gathered}
$$

$$
\sigma_{3}^{2}(850 \mathrm{~nm})=3.3253 \cdot\left(0.057 k^{2} L D^{5 / 3}\right) C_{n}^{2}
$$

\subsection{EXPERIMENTAL SETUP FOR TILT CORRECTION}

The experimental setup used for the correction of atmospheric wavefront tilt is shown in Figure 2. Two beams in the transmitter: one at $1550 \mathrm{~nm}$-wavelength for the tracking or correcting signal and another for the quantum channel at $850 \mathrm{~nm}$ were used. Lenses $\mathrm{L}_{1}$ and $\mathrm{L}_{3}$ for the $850 \mathrm{~nm}$ wavelength beam and $\mathrm{L}_{2}$ and $\mathrm{L}_{3}$ for $1550 \mathrm{~nm}$ wavelength were used to obtain two identical $50 \mathrm{~mm}$ diameter beams. They were then spectrally combined using a Dichroic Mirror (DM) and mounted over a two-motor gimbal platform that provided elevation and azimuth $\mu$ rad precision movements.

In the receiver, we used a $25.4 \mathrm{~cm}$ Schmidt-Cassegrain telescope to collect both tracking and quantum signals, and a Dichroic Mirror (DM) to spectrally discriminate them and direct them to their corresponding channels. The quantum channel included a lateral-effect PSD and an optical fiber to record the position of the beam and the optical power, respectively, before and after correcting. The quantum channel is a simplification of the QKD quantum receiver, since the characterization of the performance of the correcting system was the main objective. The transmitter and receiver were located at two different buildings separated by a distance of 300 meters in CSIC campus.

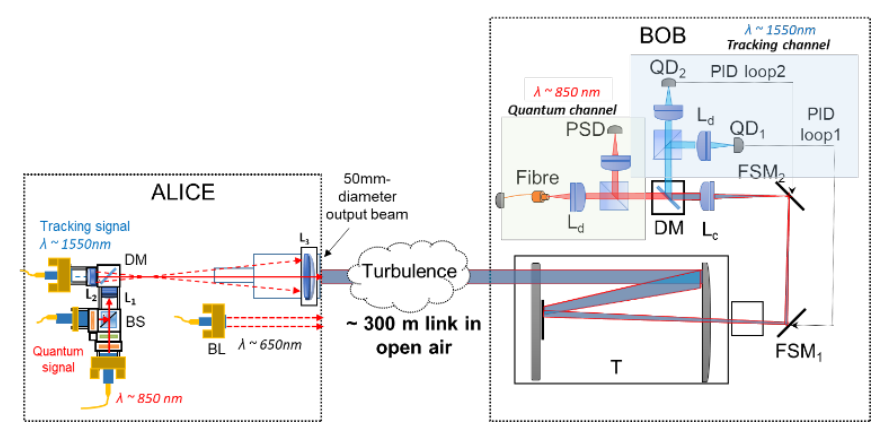

Figure 2. Transmitter and receiver used for characterising the wavefront tilt correction. DM: Dichroic mirror; BL: beacon laser; BS: 50/50 beamsplitter; PSD: lateral-effect position sensitive detector; QD: quadrant detector; BS: beamsplitter; FSM: fast steering mirror; Lc: collimating lens; Ld: detector lens; T: SchmidtCassegrain telescope. 
The spot focal wander $\sqrt{\left\langle r_{a}^{2}\right\rangle}$ was measured at the focal plane of the receiver by the PSD to obtain the angle of arrival $\sqrt{\left\langle\beta_{a}^{2}\right\rangle}$ before and after correcting tilt throughout a measurement of 9 hours. Tilt correction used two PIDs loops [3]. The data before correction was used to calculate the refractive index structure parameter $C_{n}^{2}$ through equation (6). This data along with the long-term beam diameter ( $\sim 60 \mathrm{~mm}$ for the maximum turbulence measured) was then used to calculate the experimental residual error $\sigma_{3}^{2}$ by equation (19) and was compared to the theoretical values taken from equations (20) and (23) (see Figure 3).

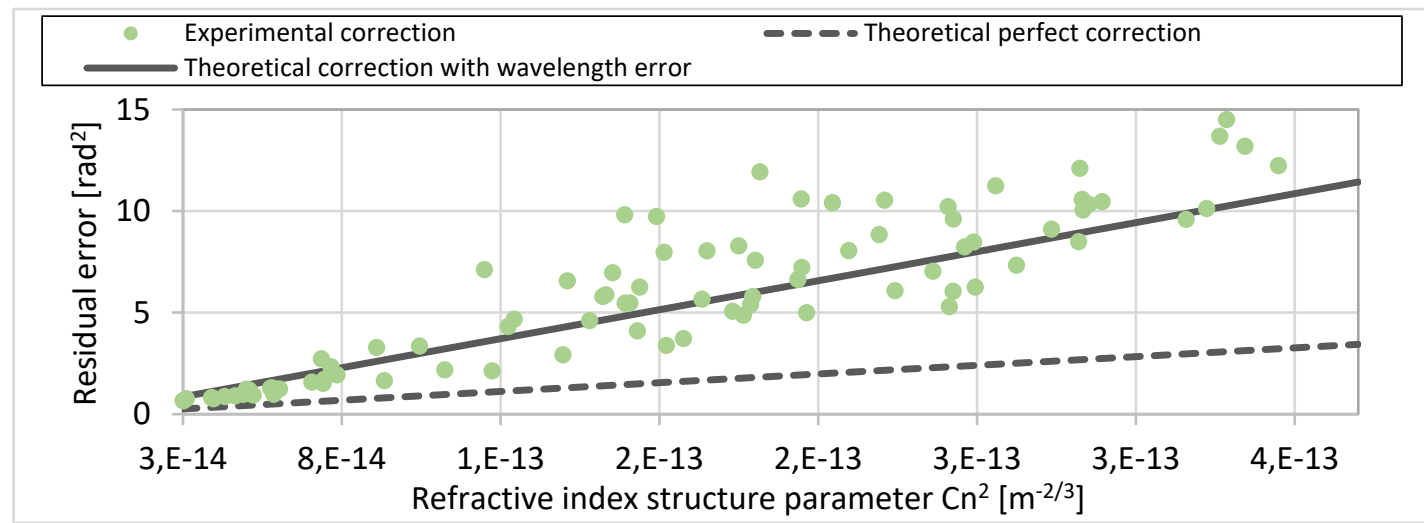

Figure 3: Residual error after correcting tilt. The theoretical values are shown for two cases: with and without considering the error introduced by using a different wavelength for correcting. In circles are the experimental values of the residual error after correcting.

It can be seen from the theoretical values of the residual error in Figure 3 that this increases with the turbulent regime, even when correcting in the ideal case, which corresponds to the correction being performed with a signal of the same wavelength as the data signal. This is never the case for quantum communications though, since there would be no way to discriminate the quantum and tracking signals. Since using a different wavelength for the tracking increases the error, the tracking and quantum channels should be as close in wavelength as possible to reduce this error. Nevertheless, the achieved correction is in very good agreement with the maximum theoretical performance that the system can achieve. The obtained reduction of the turbulent deviations in the focal area of the receiver correspond to an equivalent reduction in the QBER of more than $80 \%$ in strong turbulent conditions $\left(C_{n}^{2} \sim 10^{-13} \mathrm{~m}^{-2 / 3}\right)$.

\subsection{CONCLUSIONS}

Efficient correction of atmospheric effects such as wavefront tilt is essential for the success future quantum wireless networks. A system for tilt correction for free-space QKD systems has been designed and tested. The residual error after correcting tilt was measured experimentally, and compared to the theoretical prediction to assess the quality of the correction, which was found to be in close agreement to a perfect theoretical correction. The experimental values correspond to a reduction of the QBER of more than $80 \%$, since reducing the focal area of beam deviations in the receiver decreases the photon noise contribution due to solar stray photons. This opens the door to successful QKD in realistic conditions over short links typical from wireless telecommunication networks.

\section{ACKNOWLEDGMENTS}

This work was supported by Ministerio de Economía y Competitividad, Grant TEC2015-70406-R (MINECO/FEDER, UE), Fondo Social Europeo through Programa Operativo de Empleo Juvenil. We also acknowledge support from CSIC Research Platform on Quantum Technologies PTI-001.

\section{REFERENCES}

[1] L. C. Andrews and R. L. Phillips, "Laser Beam Propagation Through Random Media". SPIE Optical Engineering Press, Bellinngham, WA, USA, 2005.

[2] R. J. Noll, "Zernike polynomials and atmospheric turbulence", J. Opt. Soc. Am., vol. 66, No. 3, pp. 207 $211,1976$.

[3] A. Ocampos-Guillén, N. Denisenko, V. Fernandez, "Optimising the interconnection of free space to fibre quantum networks" Quantum Technology International Conference, Proc. EPJ-Web of conferences, Vol 198, 00007, 2019, DOI: https://doi.org/10.1051/epjconf/201919800007 